\title{
Fast and Robust Reconstruction for Fluorescence Molecular Tomography via $L_{1-2}$ Regularization
}

\author{
Haibo Zhang, Guohua Geng, Xiaodong Wang, Xuan Qu, Yuqing Hou, and Xiaowei He \\ School of Information Sciences and Technology, Northwest University, Xian, Shaanxi 710027, China \\ Correspondence should be addressed to Guohua Geng; ghgeng@nwu.edu.cn and Xiaowei He; hexw@nwu.edu.cn
}

Received 19 June 2016; Revised 10 September 2016; Accepted 25 September 2016

Academic Editor: Jun Zhang

Copyright (c) 2016 Haibo Zhang et al. This is an open access article distributed under the Creative Commons Attribution License, which permits unrestricted use, distribution, and reproduction in any medium, provided the original work is properly cited.

\begin{abstract}
Sparse reconstruction inspired by compressed sensing has attracted considerable attention in fluorescence molecular tomography (FMT). However, the columns of system matrix used for FMT reconstruction tend to be highly coherent, which means $L_{1}$ minimization may not produce the sparsest solution. In this paper, we propose a novel reconstruction method by minimization of the difference of $L_{1}$ and $L_{2}$ norms. To solve the nonconvex $L_{1-2}$ minimization problem, an iterative method based on the difference of convex algorithm (DCA) is presented. In each DCA iteration, the update of solution involves an $L_{1}$ minimization subproblem, which is solved by the alternating direction method of multipliers with an adaptive penalty. We investigated the performance of the proposed method with both simulated data and in vivo experimental data. The results demonstrate that the DCA for $L_{1-2}$ minimization outperforms the representative algorithms for $L_{1}, L_{2}, L_{1 / 2}$, and $L_{0}$ when the system matrix is highly coherent.
\end{abstract}

\section{Introduction}

Fluorescence molecular tomography (FMT) has become a promising molecular imaging modality since it has the ability to provide localization and quantitative analysis of the fluorescent probe for preclinical research $[1,2]$. However, FMT reconstruction suffers from high ill-posedness due to the insufficiency of external measurements, which is caused by high absorption and scattering in photon propagation through biological tissues [3].

To alleviate the ill-posedness of FMT, some a priori information, such as anatomical information, optical properties, permissible region, and sparsity of target distribution, has been successfully incorporated in FMT reconstruction [4-7]. In addition, many regularization techniques have also been devoted to get an accurate and stable solution. Conventionally, $L_{2}$ norm regularizer is a common penalty term in spite of its over-smoothness and results with lower resolution [8]. Another common regularizer is $L_{0}$ norm, which is nondeterministic polynomial (NP) hard and can be solved by a greedy approach such as the orthogonal matching pursuit (OMP) [9]. Inspired by compressive sensing (CS) theory, the $L_{1}$ norm regularizer as the convex relaxation of
$L_{0}$ has become a widely used sparsity-inducing norm for FMT reconstruction [10-13]. However, $L_{1}$ norm regularizer is not always providing the sparsest solution for the inverse problem of FMT [14]. This gives way to nonconvex $L_{p}(0<$ $p<1)$ norm regularizer, which has been applied to optical tomography and was found to have better results than $L_{1}$ does [15]. Some comparative studies show that nonconvex $L_{p}$ $(0<p<1)$ norm regularizer with $p$ near $1 / 2$ performs the best result among regularizers of $L_{p}(0<p<1)$ norm [16].

Recently, a new nonconvex regularizer named $L_{1-2}$ has been proposed and produced better solution than $L_{p}(p=$ $1 / 2$ ) norm regularizer when the sensing matrix was large and highly coherent $[17,18]$. The Magnetic Resonance Imaging (MRI) image recovery tests have also indicated that $L_{1-2}$ norm regularizer outperforms $L_{1 / 2}$ and $L_{1}$ for highly coherent matrix [17]. Meanwhile, the columns of system matrix used for FMT reconstruction are also highly coherent with the finite element computing framework [19].

In this paper, new $L_{1-2}$ norm regularization was proposed to improve the FMT imaging. In our method, a difference of convex algorithm (DCA) was presented to solve the nonconvex $L_{1-2}$ minimization problem. And the alternating direction method of multipliers (ADMM) with an adaptive penalty was 
used to solve the subproblem with fast convergence for each DCA iteration. The performance of the proposed method was validated with simulated data and in vivo experimental data.

The outline of this paper is as follows. Section 2 elaborates the forward model and $L_{1-2}$ norm regularization algorithm. Section 3 demonstrates the feasibility and effectiveness of the method with both simulated data and in vivo experimental data. Finally, we conclude the paper and discuss relevant issues in Section 4.

\section{Methods}

2.1. Light Propagation Model. As an approximation to Radiative Transfer Equation (RTE), the Diffusion Approximation associated with Robin boundary conditions has been widely used for modeling the light transportation in biological tissues [20, 21]. For steady-state FMT with point excitation sources, the coupled diffusion equations can be presented as follows:

$$
\begin{aligned}
& \nabla \cdot\left(D_{\mathrm{ex}}(r) \nabla \Phi_{\mathrm{ex}}(r)\right)-\mu_{a, \mathrm{ex}}(r) \Phi_{\mathrm{ex}}=-\Theta \delta\left(r-r_{s}\right) \\
& \nabla \cdot\left(D_{\mathrm{em}}(r) \nabla \Phi_{\mathrm{em}}(r)\right)-\mu_{a, \mathrm{em}}(r) \Phi_{\mathrm{em}} \\
& \quad=-\Phi_{\mathrm{ex}}(r) \eta \mu_{a f}(r)
\end{aligned}
$$

where subscript ex and em denote excitation light and emission light, respectively. $r \in \Omega$ is the domain under consideration. $D_{\mathrm{ex}}=1 / 3\left(\mu_{a, \mathrm{ex}}+\mu_{s, \mathrm{ex}}^{\prime}\right)$ and $D_{\mathrm{em}}=1 / 3\left(\mu_{a, \mathrm{em}}+\right.$ $\left.\mu_{s, \mathrm{em}}^{\prime}\right)$ are diffusion coefficients with $\mu_{a, \mathrm{ex}}, \mu_{a \text {,em }}$ as absorption coefficients for excitation and emission wavelengths, $g$ is the anisotropy parameter, and $\mu_{s, \mathrm{ex}}^{\prime}, \mu_{s, \mathrm{em}}^{\prime}$ are the reduced scattering coefficients. $\Phi_{\mathrm{ex}}$ and $\Phi_{\mathrm{em}}$ denote the photon density. $\eta \mu_{a f}$ is the unknown fluorescent yield to be reconstructed. Using the finite elements method (FEM), (1) can be linearly discretized as follows:

$$
\begin{gathered}
K_{\mathrm{ex}} \Phi_{\mathrm{ex}}=b_{\mathrm{ex}} \\
K_{\mathrm{em}} \Phi_{\mathrm{em}}=F X,
\end{gathered}
$$

where $K_{\mathrm{ex}}$ and $K_{\mathrm{em}}$ denote the system matrix at excitation and emission wavelengths, respectively. The symmetric matrix $F$ is obtained by discretizing the unknown fluorescent yield distribution. The final linear relationship between the unknown fluorescence yield $x$ and the measured surface data $\phi$ can be obtained as follows:

$$
A x=\phi
$$

where $A$ is $M \times N$ linear system matrix which is large-sized and ill-posed.

2.2. Inverse Reconstruction of FMT by DCA- $L_{1-2}$ Algorithm. The CS theory provides sufficient conditions for the exact recovery of the sparse signals from limited number of measurements. One commonly used concept is the mutual coherence $[17,22]$ which is defined as

$$
\mu(A)=\max _{i \neq j} \frac{\left|a_{p}^{T} \cdot a_{q}\right|}{\left\|a_{p}\right\|_{2} \cdot\left\|a_{q}\right\|_{2}},
$$

where $a_{p}$ and $a_{q}$ are different columns of $A$. The mutual coherence of system matrix $A$ derived by FEM method is always as high as above $90 \%$ [19]. In the highly coherent regime of CS, $L_{p}(p=1 / 2)$ and $L_{1-2}$ norm regularizers are expected to yield the sparest solution that $L_{1}$ regularization always fails to $[17,18]$.

Recently, a DCA- $L_{1-2}$ algorithm was proposed and theoretical properties of $L_{1-2}$ minimization have been proved in papers $[17,23]$. Considering the advantages of $L_{1-2}$ minimization, we converted linear matrix equation (3) into the following unconstrained optimization problem:

$$
\min _{x \in \mathbb{R}^{n}} \frac{1}{2}\|A x-\phi\|_{2}^{2}+\lambda\left(\|x\|_{1}-\|x\|_{2}\right),
$$

where $\lambda>0$ is a regularization parameter which is usually empirically selected and $\|x\|_{1}-\|x\|_{2}$ denotes the $L_{1-2}$ regularization operator.

To resolve minimization problem (5), the difference of convex algorithm (DCA) [24] which is a descent method without line search was used. Equation (5) can be decomposed into DC decomposition as $F(x)=G(x)-H(x)$, where

$$
\begin{aligned}
& G(x)=\frac{1}{2}\|A x-\phi\|_{2}^{2}+\lambda\|x\|_{1}, \\
& H(x)=\lambda\|x\|_{2} .
\end{aligned}
$$

In (6), $\|x\|_{2}$ is differentiable with gradient $x /\|x\|_{2}$. An iterative scheme was used to solve $F(x)$ as follows:

$$
\begin{aligned}
x^{n+1}= & \arg \min _{x \in \mathbb{R}^{n}} \frac{1}{2}\|A x-\phi\|_{2}^{2}+\lambda\|x\|_{1} \\
& -\left\langle x-x^{n}, \frac{x^{n}}{\left\|x^{n}\right\|_{2}}\right\rangle .
\end{aligned}
$$

In each DCA iteration, there is a $L_{1}$-regularized convex subproblem that needs to be solved:

$$
\min _{x \in \mathbb{R}^{n}} \frac{1}{2} x^{T}\left(A^{T} A\right) x+\left(x^{T} \phi+\lambda \frac{x^{n}}{\left\|x^{n}\right\|_{2}}\right)^{T} x+\lambda\|x\|_{1} .
$$

We use the augmented Lagrangian method and transform (8) into the following:

$$
\begin{aligned}
L_{\delta}(x, y, u)= & \frac{1}{2} x^{T}\left(A^{T} A\right) x+\left(A^{T} \phi+\lambda \frac{x^{n}}{\left\|x^{n}\right\|_{2}}\right)^{T} x \\
& +\lambda\|y\|_{1}+u^{T}(x-y)+\frac{\delta}{2}\|x-y\|_{2}^{2} .
\end{aligned}
$$

The subproblem is solved by minimizing $L_{\delta}$ with respect to $x$, minimizing $L_{\delta}$ with respect to $y$, and updating $u$ successively. In order to solve (9) with a fast speed of convergence, an ADMM strategy with an adaptive penalty [25] was utilized as follows:

$$
\begin{aligned}
& x^{k+1}=\arg \min _{x} L_{\delta_{k}}\left(x, y^{l}, u^{l}\right), \\
& y^{k+1}=\arg \min _{z} L_{\delta_{k}}\left(x^{l+1}, y, u^{l}\right), \\
& u^{k+1}=u^{l}+\delta_{k}\left(x^{l+1}-y^{l+1}\right), \\
& \delta_{k+1}=\min \left(\delta_{\max }, \rho \delta_{k}\right) .
\end{aligned}
$$




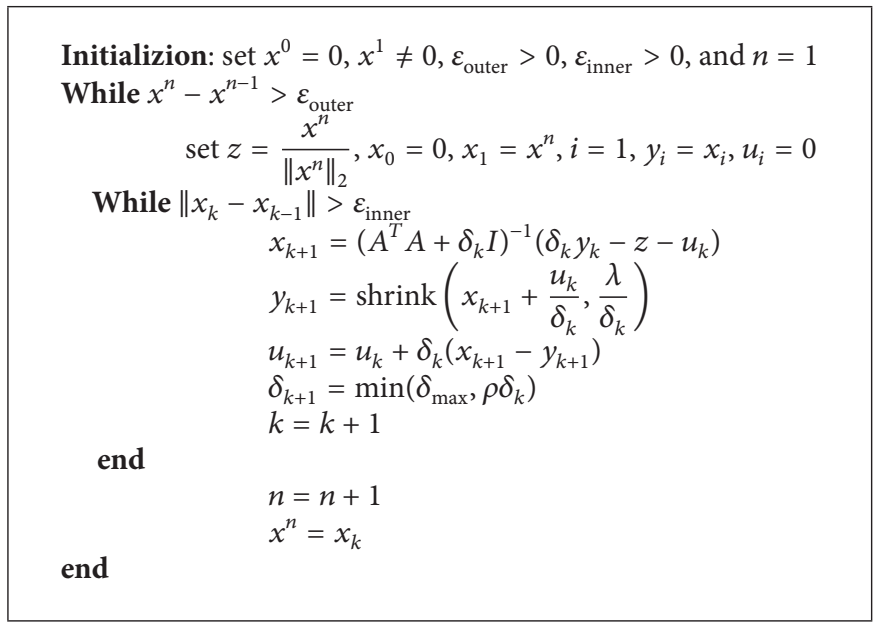

Algorithm 1: DCA- $L_{1-2}$ algorithm for FMT.

In the above iterations, the update of $y$ is based on the soft-thresholding operator, where

$$
(S(x, r))_{i}=\operatorname{sgn}\left(x_{i}\right) \max \left\{\left|x_{i}\right|-r, 0\right\} .
$$

Meanwhile, the penalty $\delta$ was updated as an adaptive form as follows:

$$
\delta_{k+1}=\min \left(\delta_{\max }, \rho \delta_{k}\right) \text {, }
$$

where $\delta_{\max }$ is an upper bound of $\left\{\delta_{k}\right\}$ and $\rho$ is defined as follows:

$$
\rho= \begin{cases}\rho_{0}, & \text { if } \frac{\delta\left\|y^{k+1}-y^{k}\right\|}{x^{k+1}}<\varepsilon \\ 1, & \text { otherwise, }\end{cases}
$$

where $\rho_{0} \geq 1$ is a constant.

Algorithm 1 presents the iterative process of DCA- $L_{1-2}$ algorithm for FMT reconstruction. To begin with the iteration, the initial value $x^{1}$ was set as $L_{1}$ subproblem.

\section{Experiments and Results}

In this section, the simulations both on $3 \mathrm{D}$ digital mouse model and in vivo experiments were used to demonstrate the potential and feasibility of the DCA- $L_{1-2}$ algorithm for FMT reconstruction. To investigate the performance of DCA- $L_{1-2}$ algorithm, four representative regularizers, including $L_{1 / 2}$, $L_{1}, L_{2}$, and $L_{0}$, were used for a systematical comparison. More specifically, iterative reweighted least squares algorithm (IRLS- $L_{1 / 2}$ ) [26], incomplete variables truncated conjugate gradient algorithm (IVTCG- $L_{1}$ ) [27], Tikhonov regularization algorithm (Tikhonov- $L_{2}$ ) [28], and OMP algorithm [29] were compared with the DCA- $L_{1-2}$ algorithm, respectively, as the corresponding method for the above regularizers.

The qualities of reconstruction results are quantitatively evaluated in terms of the absolute location error (LE) [3], reconstructed fluorescent yield (Recon. FY) [3], normalized root mean square error (NRMSE) [16], the percentage of
TABLE 1: Optical parameters for the heterogeneous model.

\begin{tabular}{lcccc}
\hline Organs & $\mu_{a, \mathrm{ex}}\left(\mathrm{mm}^{-1}\right)$ & $\mu_{s, \mathrm{ex}}^{\prime}\left(\mathrm{mm}^{-1}\right)$ & $\mu_{a, \mathrm{em}}\left(\mathrm{mm}^{-1}\right)$ & $\mu_{s, \mathrm{em}}^{\prime}\left(\mathrm{mm}^{-1}\right)$ \\
\hline Muscle & 0.0052 & 1.08 & 0.0068 & 1.03 \\
Heart & 0.0083 & 1.01 & 0.0104 & 0.99 \\
Lungs & 0.0133 & 1.97 & 0.0203 & 1.95 \\
Liver & 0.0329 & 0.70 & 0.0176 & 0.65 \\
Kidneys & 0.0660 & 2.25 & 0.0380 & 2.02 \\
Stomach & 0.0114 & 1.74 & 0.0070 & 1.36 \\
\hline
\end{tabular}

nonzero coefficient (PNZ) [16], and time cost. The experiment codes were written in MATLAB and were performed on a desktop computer with $3.40 \mathrm{GHz}$ Intel ${ }^{\circledR} \mathrm{Xeon}^{\circledR}$ Processor E3-1231 and 12 G RAM.

3.1. Numerical Simulation Experiments. A $33 \mathrm{~mm}$ height torso extracted from a 3D mouse atlas was utilized to simulate the heterogeneity of biological tissues [30]. Figure 1(a) shows the mouse model with six organs. Table 1 lists the specific optical parameters. A cylinder with a radius of $0.8 \mathrm{~mm}$ and a height of $1.6 \mathrm{~mm}$ was positioned at $17.8,6.6$, and $16.4 \mathrm{~mm}$ to mimic the fluorescent target. The actual fluorescent yield was set to be $0.05 \mathrm{~mm}^{-1}$. For excitation, we used 18 excitation sources being located on the plane of $Z=16.4 \mathrm{~mm}$ as shown in Figure 2(a). The surface data on the opposite side with a $120^{\circ}$ field of view (FOV) were measured for each excitation source. A total of 18 datasets were assembled for the subsequent reconstruction process.

The forward FEM mesh was discretized into 24231 nodes and 128300 tetrahedral elements. Meanwhile, the FEM mesh for inverse reconstruction was discretized into 2601 nodes and 12752 tetrahedral elements. The mutual coherence of the system matrix for inverse reconstruction was $99.96 \%$.

Figures 1(b)-1(f) present a comparison of reconstruction results with $3 \mathrm{D}$ views for single fluorescent target. The corresponding $2 \mathrm{D}$ section-views on the excitation plane are demonstrated in Figures 2(b)-2(f). Table 2 gives the quantitative results of the five regularization methods. 


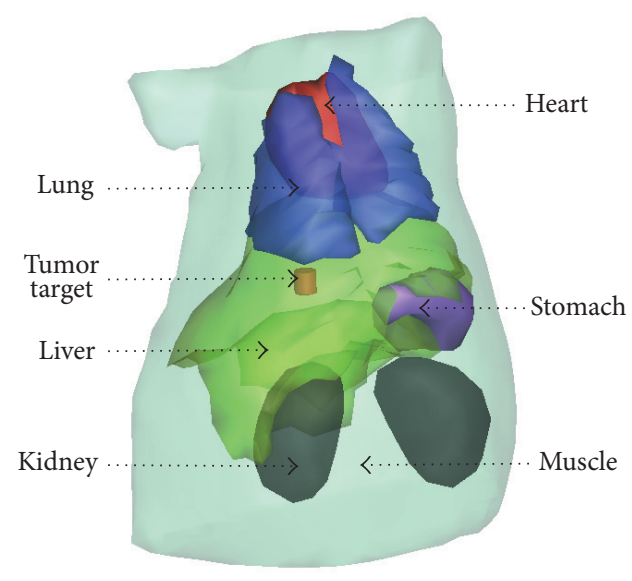

(a) 3D mouse with single target

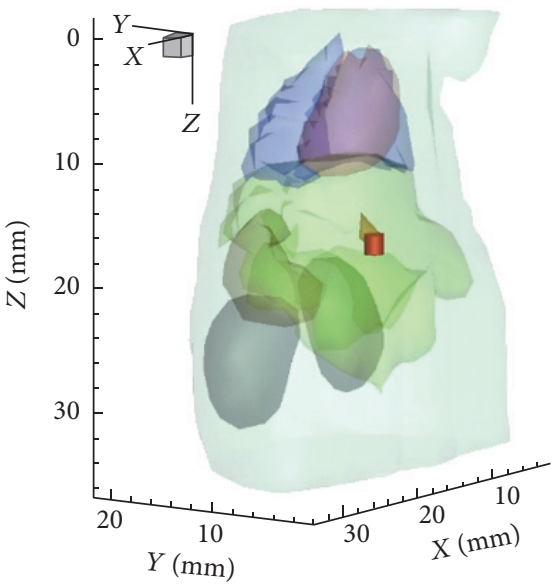

(c) $L_{1 / 2}$

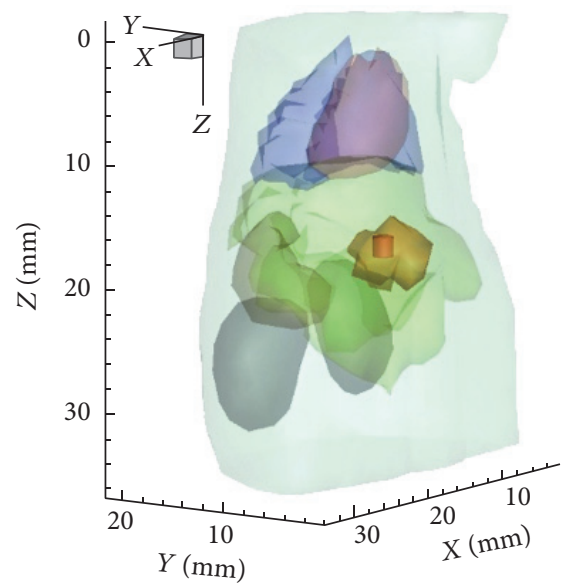

(e) $L_{2}$

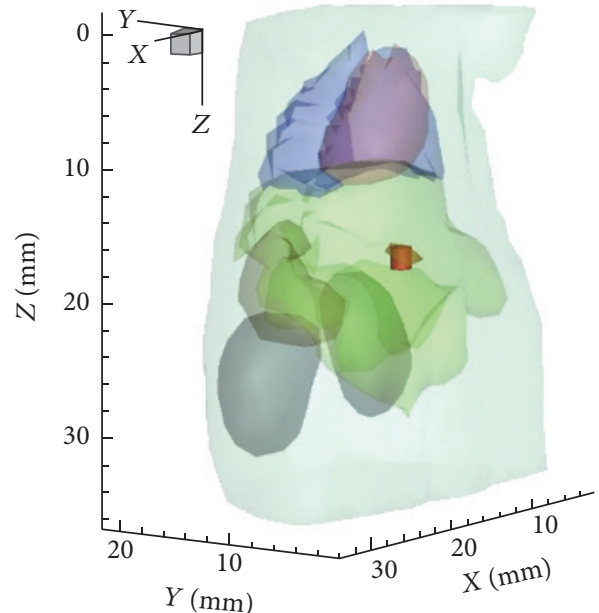

(b) $L_{1-2}$

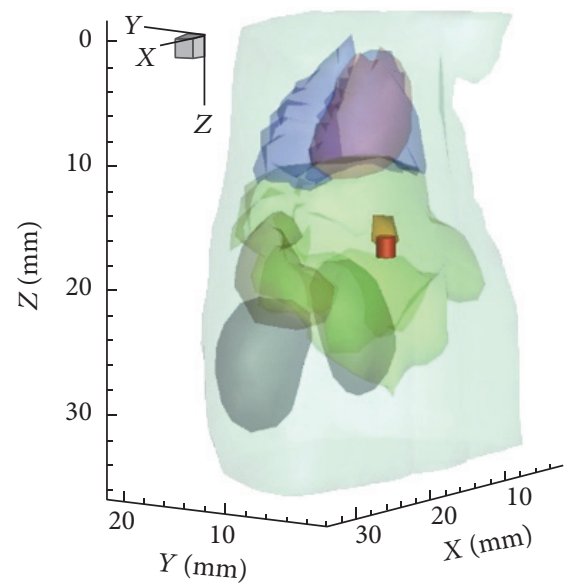

(d) $L_{1}$

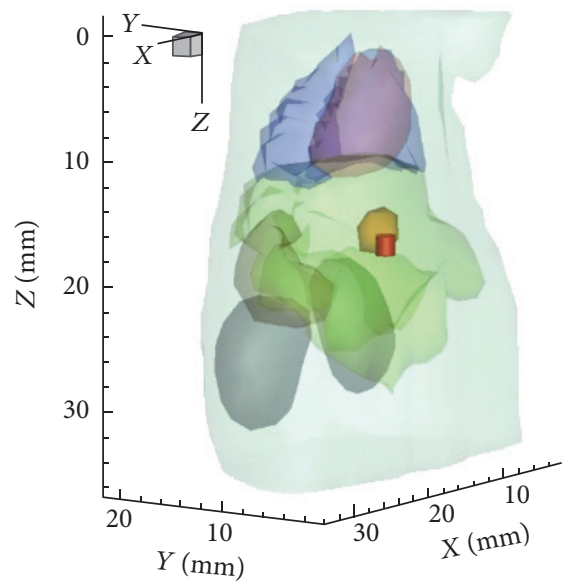

(f) $L_{0}$

FIGURE 1: The mouse model and the 3D views of the reconstructed results. (a) The mouse model with single target. (b-f) The 3D views of the reconstructed results where the red cylinder is the real fluorescent target. 


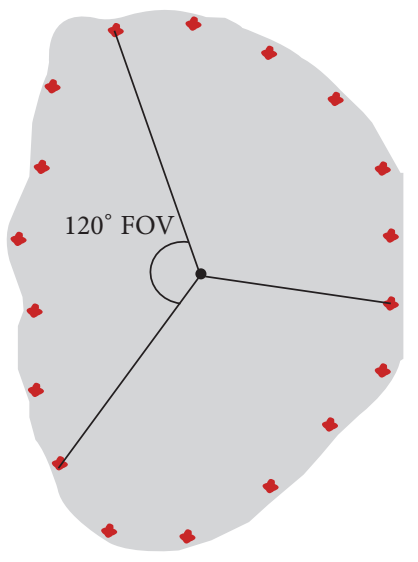

(a) The plane of excitation source of $z=16.4$

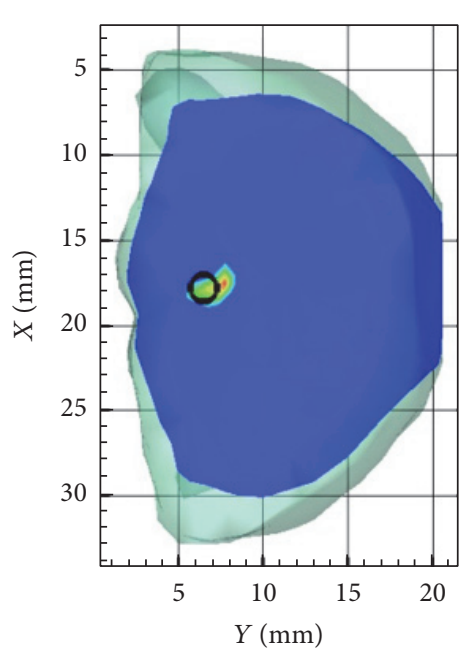

(c) $L_{1 / 2}$

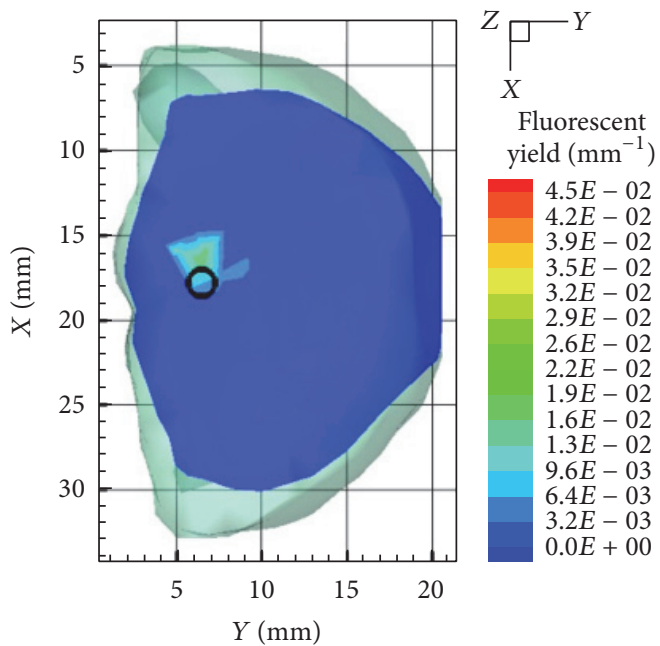

(e) $L_{2}$

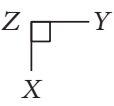

Fluorescent yield $\left(\mathrm{mm}^{-1}\right)$

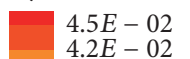

$4.2 E-02$

$3.9 E-02$
$3.5 E-02$

$3.5 E-02$
$3.2 E-02$

$2.9 E-02$

$2.6 E-02$

$2.2 E-02$

$1.9 E-02$

$1.6 E-02$

$1.3 E-02$

$9.6 E-03$

$6.4 E-03$

$3.2 E-03$

$0.0 E+00$

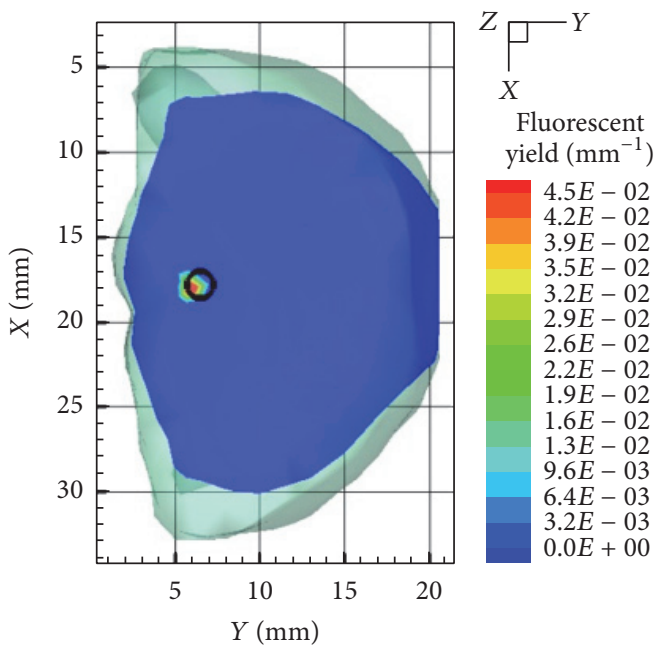

(b) $L_{1-2}$

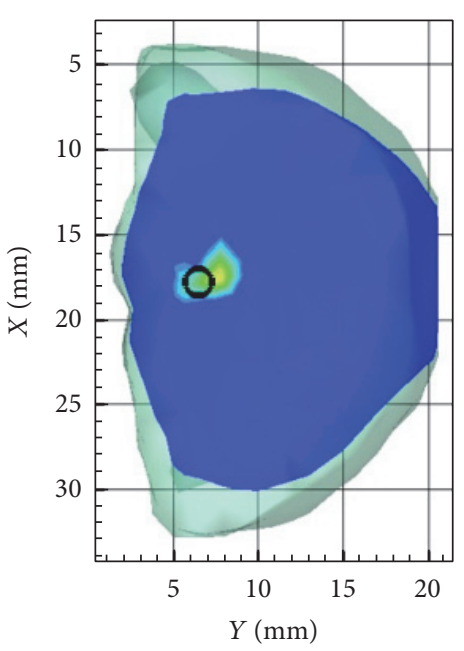

${ }^{Z}{ }_{X} Y$

Fluorescent yield $\left(\mathrm{mm}^{-1}\right)$

$4.5 E-02$
$4.2 E-02$

$3.9 E-02$

$3.9 E-02$
$3.5 E-02$

$3.2 E-02$

$2.9 E-02$

$2.6 E-02$

$2.2 E-02$

$1.9 E-02$

$1.6 E-02$

$1.3 E-02$

$9.6 E-03$

$6.4 E-03$

$3.2 E-03$

$0.0 E+00$

(d) $L_{1}$

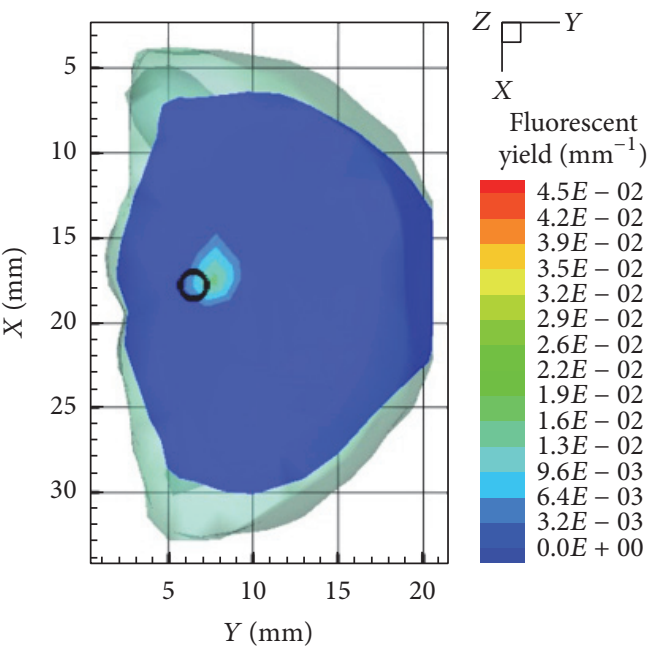

(f) $L_{0}$

Figure 2: The plane of 18 excitation sources and the $2 \mathrm{D}$ views $(Z=16.4 \mathrm{~mm})$ of the reconstructed results. (a) The plane of 18 excitation sources with $120^{\circ}$ FOV. (b-f) The $2 \mathrm{D}$ views $(Z=16.4 \mathrm{~mm}$ ) of the reconstructed results by six comparative methods. The black circle denotes real position of fluorescent target. 
TABLE 2: Quantitative results in single target reconstruction experiment.

\begin{tabular}{lccccc}
\hline Methods & LE $(\mathrm{mm})$ & Recon. FY $\left(\mathrm{mm}^{-1}\right)$ & NRMSE $(\%)$ & PNZ (\%) & Time (s) \\
\hline DCA- $L_{1-2}$ & 0.436 & 0.039 & 19 & 1.15 & 1.32 \\
IRLS- $L_{1 / 2}$ & 0.668 & 0.032 & 29 & 1.86 & 1.67 \\
IVTCG- $L_{1}$ & 1.279 & 0.005 & 67 & 7.47 & 31.74 \\
Tikhonov- $L_{2}$ & 2.169 & 0.004 & 63 & 11.96 & 9.83 \\
OMP & 1.135 & 0.004 & & & 1.21 \\
\hline
\end{tabular}

TABLE 3: The results of DCA- $L_{1-2}$ with different excitation sources.

\begin{tabular}{lcccc}
\hline The number of excitation sources & LE $(\mathrm{mm})$ & Recon. FY $\left(\mathrm{mm}^{-1}\right)$ & NRMSE $(\%)$ & PNZ $(\%)$ \\
\hline 18 & 0.436 & 0.039 & 19 & 1.15 \\
12 & 0.497 & 0.034 & 21 & 1.28 \\
8 & 0.518 & 0.029 & 23 & 1.67 \\
4 & 0.614 & 0.014 & 36 & 2.34 \\
\hline
\end{tabular}

TABLE 4: Impact of Gaussian noise on DCA- $L_{1-2}$.

\begin{tabular}{lccccc}
\hline Noise level (\%) & LE $(\mathrm{mm})$ & Recon. FY $\left(\mathrm{mm}^{-1}\right)$ & NRMSE $(\%)$ & PNZ (\%) & Time (s) \\
\hline 5 & 0.436 & 0.039 & 19 & 1.15 & 1.67 \\
15 & 0.437 & 0.039 & 19 & 1.15 & 1.15 \\
25 & 0.437 & 0.038 & 20 & 1.73 & 1.33 \\
35 & 0.514 & 0.038 & 20 & 1.78 \\
\hline
\end{tabular}

As shown in Figures 1(b)-1(f), Figures 2(b)-2(f), and Table 2, reconstruction results of $L_{1-2}$ and $L_{1 / 2}$ were remarkable. Compared to the other three methods, results of DCA$L_{1-2}$ and IRLS- $L_{1 / 2}$ have fewer artifacts, lower LE, lower NRMSE, and lower PNZ. Meanwhile, the Recon. FY by DCA$L_{1-2}$ and IRLS- $L_{1 / 2}$ were closer to $0.05 \mathrm{~mm}^{-1}$. The proposed DCA- $L_{1-2}$ completely outperforms the other methods, except for a slightly larger time consumption compared to OMP.

Generally speaking, the quality of FMT reconstruction is influenced by the number of excitation sources. To test the stability of the algorithm, different numbers of excitation sources were used for reconstruction. Table 3 gives the corresponding reconstructed results with 18, 12, 8, and 4 . Obviously, the decreased number of excitation sources leads to significant reduction of measurements. Nevertheless, the results of DCA- $L_{1-2}$ are generally satisfactory even in the case of 4 excitation sources.

The quality of reconstructed results is sensitive to measurement noise because of the severe ill-condition of system matrix. For stability test, four different levels of Gaussian noise $(5 \%, 15 \%, 25 \%$, and $35 \%)$ were added to the synthetic measurements. Table 4 shows the reconstruction results under 4 different noise levels. It shows that the DCA- $L_{1-2}$ algorithm is quite resilient with Gaussian noise.

3.2. In Vivo Evaluation with Implanted Fluorophore. The performance of DCA- $L_{1-2}$ and IRLS- $L_{1 / 2}$ was remarkably compared to the other three methods in the simulation experiments. In this section, we further evaluated the performance of the proposed algorithm with in vivo experimental data [8].
TABLE 5: Optical parameters of the mouse model at $670 \mathrm{~nm}$ and $710 \mathrm{~nm}$.

\begin{tabular}{lcccc}
\hline Organs & \multicolumn{2}{c}{$670 \mathrm{~nm}$} & \multicolumn{2}{c}{$710 \mathrm{~nm}$} \\
& $\mu_{a, \text { ex }}\left(\mathrm{mm}^{-1}\right)$ & $\mu_{s, \mathrm{ex}}^{\prime}\left(\mathrm{mm}^{-1}\right)$ & $\mu_{a, \mathrm{em}}\left(\mathrm{mm}^{-1}\right)$ & $\mu_{s, \mathrm{em}}^{\prime}\left(\mathrm{mm}^{-1}\right)$ \\
\hline Muscle & 0.075 & 0.412 & 0.043 & 0.350 \\
Heart & 0.051 & 0.944 & 0.030 & 0.870 \\
Lungs & 0.170 & 2.157 & 0.097 & 2.093 \\
Liver & 0.304 & 0.668 & 0.176 & 0.629 \\
Kidneys & 0.058 & 2.204 & 0.034 & 2.021 \\
\hline
\end{tabular}

In this experiment, an adult BALB/C mouse with a glass tube implanted into its abdomen was used. The experimental data was acquired by a hybrid FMT/Micro-CT system [8]. The glass tube $(0.6 \mathrm{~mm}$ and the height of $2.8 \mathrm{~mm})$ was filled with Cy5.5 solution (with the extinction coefficient of about $0.019 \mathrm{~mm}^{-1} \mu \mathrm{M}^{-1}$ and quantum efficiency of 0.23 at the peak excitation wavelength of $671 \mathrm{~nm}$ ) [31]. The center of the target was determined at 21.1, 27.8, and $7.4 \mathrm{~mm}$ by the Micro-CT. The fluorescent yield of Cy5.5 was $0.0402 \mathrm{~mm}^{-1}$ [32]. For reconstruction, the $\mathrm{CT}$ data was segmented into five major anatomical components, including muscle, heart, lungs, liver, and kidneys. Table 5 shows the optical properties of different organs [33].

For inverse reconstruction, the segmented mouse torso data was discretized into a mesh with 3049 nodes and 14932 tetrahedral elements. Mutual coherence of the system matrix for inverse reconstruction was $99.87 \%$. Comparison results between DCA- $L_{1-2}$ and IRLS- $L_{1 / 2}$ are shown in Table 6 and Figure 3. The $3 \mathrm{D}$ views of reconstructed results for in vivo experiments via DCA- $L_{1-2}$ are shown in Figure 4. 
TABLE 6: Quantitative results of in vivo experiments.

\begin{tabular}{lccccc}
\hline Method & LE $(\mathrm{mm})$ & Recon. FY $\left(\mathrm{mm}^{-1}\right)$ & NRMSE $(\%)$ & PNZ (\%) & Time (s) \\
\hline DCA- $L_{1-2}$ & 1.426 & 0.034 & 27 & 0.13 & 0.53 \\
IRLS- $L_{1 / 2}$ & 1.705 & 0.019 & 45 & 0.26 & 1.90 \\
\hline
\end{tabular}
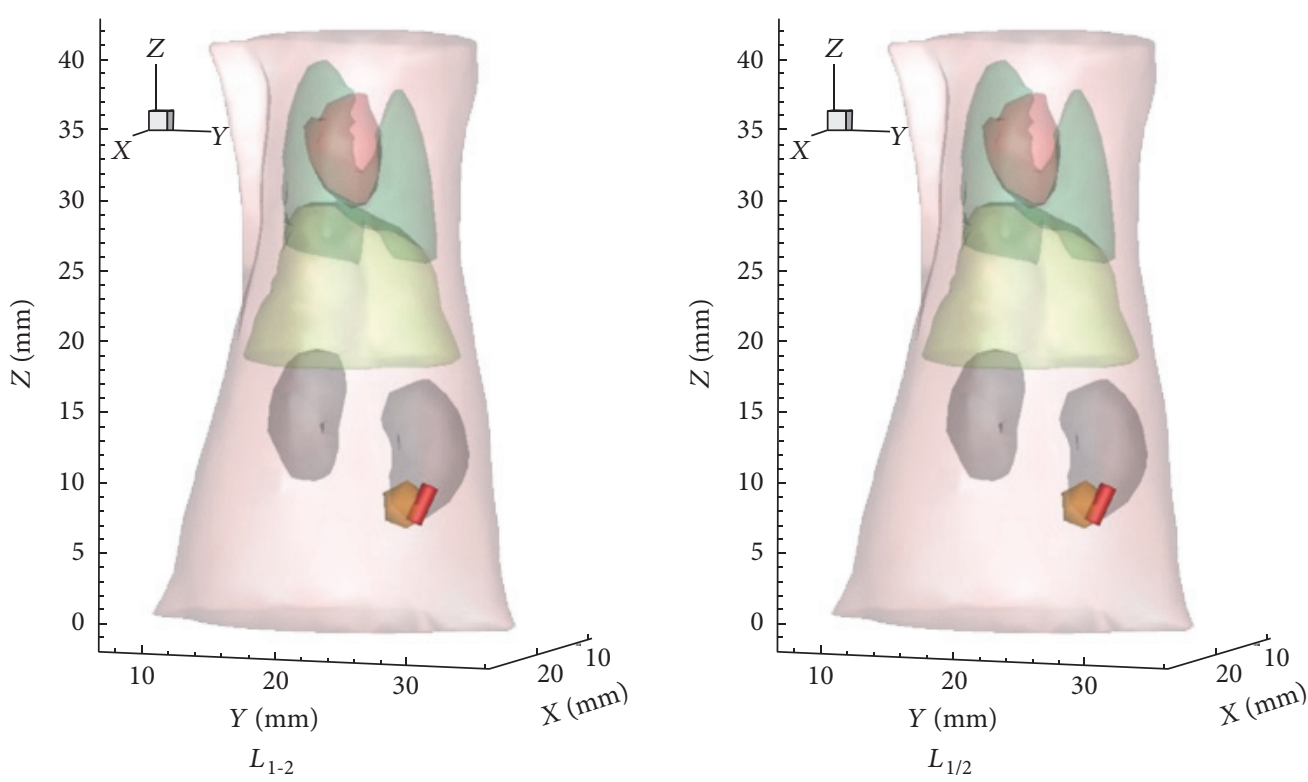

(a)
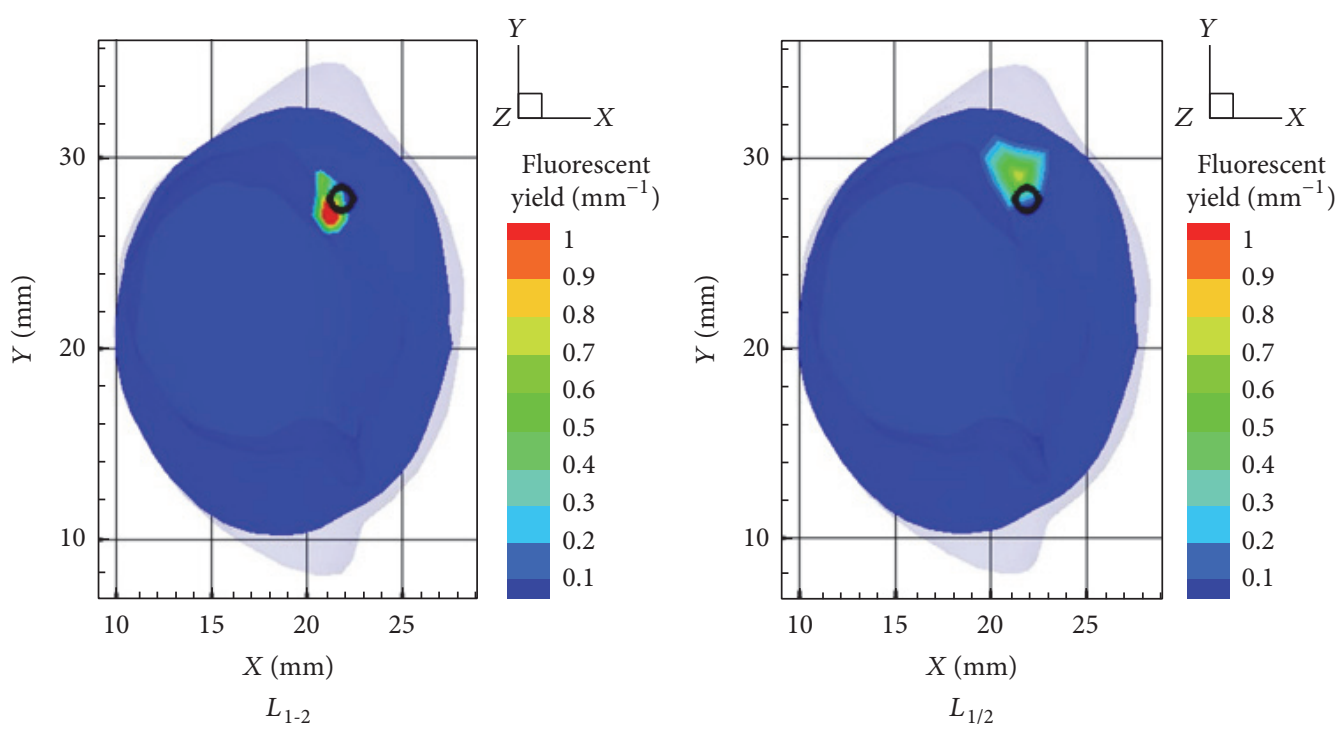

(b)

FIGURE 3: Reconstructed results in vivo experiment on adult BALB/C mouse. (a) The 3D view of the reconstructed results in which the red glass tube is the implanted fluorescent target and the green target denotes reconstructed results. (b) The $2 \mathrm{D}$ views $(Z=7.4 \mathrm{~mm})$ of the reconstructed results. The black circle denotes the real positions of fluorescent target.

\section{Discussion and Conclusion}

In this paper, novel $L_{1-2}$ norm regularization was proposed to solve the inverse problem of FMT with highly coherent system matrix. To accurately recover the small fluorescent target, an iterative method based on DCA algorithm was presented to solve the nonconvex $L_{1-2}$ minimization problem.
And the ADMM method with an adaptive penalty was used to get fast convergence for the subproblem.

Simulated data on a 3D heterogeneous mouse model and in vivo experimental data acquired by a hybrid FMT/MicroCT system were used to demonstrate the feasibility of the DCA- $L_{1-2}$ algorithm for FMT. The comparative results of single target show that the DCA- $L_{1-2}$ algorithm has better 


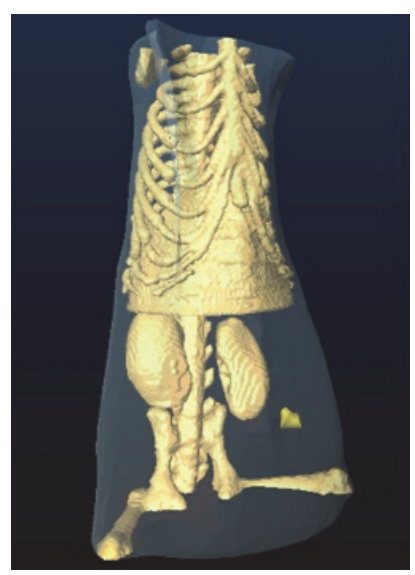

(b)

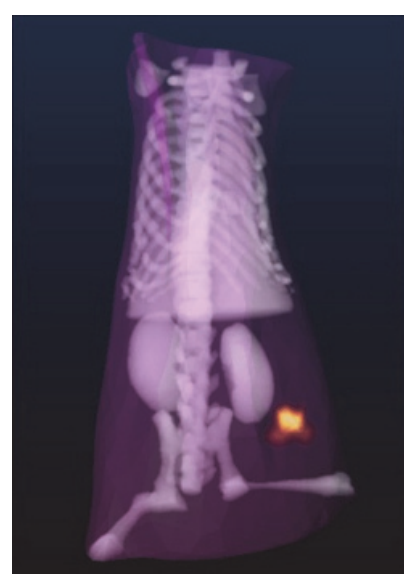

(c)

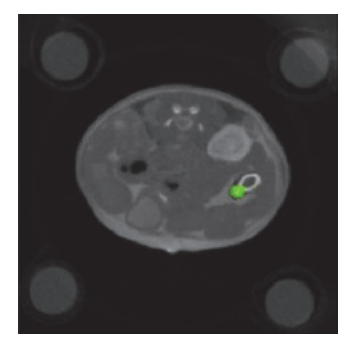

(d)

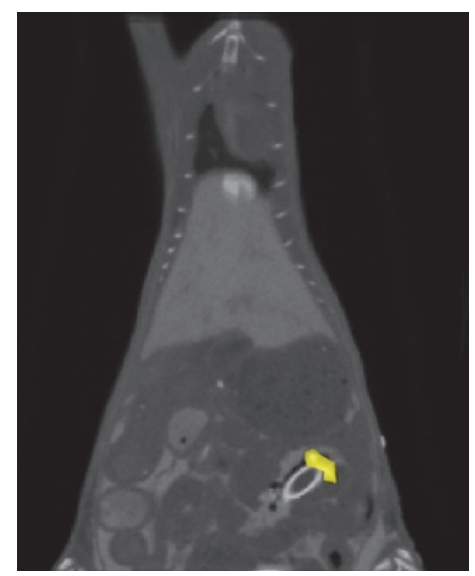

(e)

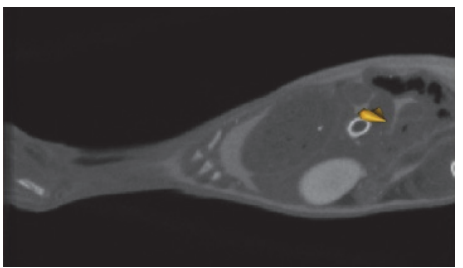

(f)

Figure 4: The reconstructed results of in vivo experiments via DCA- $L_{1-2}$. (a) $3 \mathrm{D}$ rendering of the mouse. (b) The reconstructed result in $3 \mathrm{D}$ view. (c) The photon density distribution of the reconstructed result in $3 \mathrm{D}$ view. (d-f) The $2 \mathrm{D}$ views of the overlapped result with corresponding CT slices.

performance compared to other typical algorithms based on $L_{1 / 2}, L_{1}, L_{2}$, and $L_{0}$ norm regularizer. The robustness tests further illustrate that the DCA- $L_{1-2}$ algorithm is stable and robust to measurement noise. In addition, decreasing the number of excitation sources from 18 to 4 , DCA- $L_{1-2}$ still yields satisfactory results.

However, the reconstructed fluorescent yield of the proposed method was still smaller than the true value. So new strategies that may further improve fluorescent yield will be our future research focuses. Moreover, we will also focus on investigating the multitargets resolution and new application of $L_{1-2}$ norm regularizer in other imaging modalities in the near future.

In conclusion, both numerical experiments and in vivo experiments validated the good performance of $L_{1-2}$ regularizer for FMT. Moreover, comparative experiments indicate that $L_{1-2}$ outperforms the iterative reweighted strategies for $l_{p}$ with $p=1 / 2$ when system matrix is highly coherent.

\section{Competing Interests}

The authors declare that there is no conflict of interests regarding the publication of this paper.

\section{Acknowledgments}

The authors would like to thank the School of Life Science and Technology of Xidian University for providing experimental data acquisition system. This work was supported by the National Natural Science Foundation of China under Grant nos. 61372046 and 11571012 and the Science and Technology Plan Program in Shaanxi Province of China under Grant no. 2015KW-002.

\section{References}

[1] V. Ntziachristos, C.-H. Tung, C. Bremer, and R. Weissleder, "Fluorescence molecular tomography resolves protease activity in vivo," Nature Medicine, vol. 8, no. 7, pp. 757-760, 2002. 
[2] V. Ntziachristos, J. Ripoll, L. V. Wang, and R. Weissleder, "Looking and listening to light: the evolution of whole-body photonic imaging," Nature Biotechnology, vol. 23, no. 3, pp. 313320, 2005.

[3] X. He, F. Dong, J. Yu, H. Guo, and Y. Hou, "Reconstruction algorithm for fluorescence molecular tomography using sorted L-one penalized estimation," Journal of the Optical Society of America A: Optics, Image Science, and Vision, vol. 32, no. 11, pp. 1928-1935, 2015.

[4] X. Liu, Z. Yan, and H. Lu, "Performance evaluation of a priori information on reconstruction of fluorescence molecular tomography," IEEE Access, vol. 3, pp. 64-72, 2015.

[5] D. Wang, J. He, H. Qiao, X. Song, Y. Fan, and D. Li, "Highperformance fluorescence molecular tomography through shape-based reconstruction using spherical harmonics parameterization," PLoS ONE, vol. 9, no. 4, article e94317, 2014.

[6] S. Zhu, J. Tian, G. Yan et al., "Cone beam Micro-CT system for small animal imaging and performanceevaluation," International Journal of Biomedical Imaging, vol. 2009, Article ID 960573, 9 pages, 2009.

[7] J.-C. Baritaux, K. Hassler, and M. Unser, "An efficient numerical method for general $L_{p}$ regularization in fluorescence molecular tomography," IEEE Transactions on Medical Imaging, vol. 29, no. 4, pp. 1075-1087, 2010.

[8] H. Yi, D. Chen, W. Li et al., "Reconstruction algorithms based on $l_{1}$-norm and $l_{2}$-norm for two imaging models of fluorescence molecular tomography: a comparative study," Journal of Biomedical Optics, vol. 18, no. 5, pp. 467-472, 2013.

[9] J. Shi, X. Cao, F. Liu, B. Zhang, J. Luo, and J. Bai, "Greedy reconstruction algorithm for fluorescence molecular tomography by means of truncated singular value decomposition conversion," Journal of the Optical Society of America A, vol. 30, no. 3, pp. 437-447, 2013.

[10] K. Wang, Q. Wang, Q. Luo, and X. Yang, "Fluorescence molecular tomography in the second near-infrared window," Optics Express, vol. 23, no. 10, pp. 12669-12679, 2015.

[11] F. Yang, M. S. Ozturk, L. Zhao et al., "High-resolution mesoscopic fluorescence molecular tomography based on compressive sensing," IEEE Transactions on Bio-Medical Engineering, vol. 62, no. 1, pp. 248-255, 2015.

[12] J. Ye, Y. Du, Y. An, C. Chi, and J. Tian, "Reconstruction of fluorescence molecular tomography via a nonmonotone spectral projected gradient pursuit method," Journal of Biomedical Optics, vol. 19, no. 12, Article ID 126013, 2014.

[13] D. Zhu and C. Li, "Nonuniform update for sparse target recovery in fluorescence molecular tomography accelerated by ordered subsets," Biomedical Optics Express, vol. 5, no. 12, pp. 4249-4259, 2014.

[14] L. Zhao, H. Yang, W. Cong, G. Wang, and X. Intes, " $\mathrm{L}_{p}$ regularization for early gate fluorescence molecular tomography," Optics Letters, vol. 39, no. 14, pp. 4156-4159, 2014.

[15] D. Zhu and C. Li, "Nonconvex regularizations in fluorescence molecular tomography for sparsity enhancement," Physics in Medicine and Biology, vol. 59, no. 12, pp. 2901-2912, 2014.

[16] H. Guo, J. Yu, X. He, Y. Hou, F. Dong, and S. Zhang, "Improved sparse reconstruction for fluorescence molecular tomography with $\mathrm{L}_{1 / 2}$ regularization," Biomedical Optics Express, vol. 6, no. 5, pp. 1648-1664, 2015.

[17] P. Yin, Y. Lou, Q. He et al., "Minimization of $\ell_{1-2}$ for compressed sensing," Siam Journal on Scientific Computing, vol. 37, no. 7, pp. A536-A563, 2015.
[18] Y. Lou, P. Yin, Q. He, and J. Xin, "Computing sparse representation in a highly coherent dictionary based on difference of $L_{1}$ and $L_{2}$," Journal of Scientific Computing, vol. 64, no. 1, pp. 178196, 2015

[19] A. Jin, B. Yazici, A. Ale, and V. Ntziachristos, "Preconditioning of the fluorescence diffuse optical tomography sensing matrix based on compressive sensing," Optics Letters, vol. 37, no. 20, pp. 4326-4328, 2012.

[20] J. Feng, K. Jia, G. Yan et al., "An optimal permissible source region strategy for multispectral bioluminescence tomography," Optics Express, vol. 16, no. 20, pp. 15640-15654, 2008.

[21] S. R. Arridge, M. Schweiger, M. Hiraoka, and D. T. Delpy, "A finite element approach for modeling photon transport in tissue," Medical Physics, vol. 20, no. 2, part 1, pp. 299-309, 1993.

[22] D. L. Donoho and X. Huo, "Uncertainty principles and ideal atomic decomposition," IEEE Transactions on Information Theory, vol. 47, no. 7, pp. 2845-2862, 2001.

[23] E. Esser, Y. Lou, and J. Xin, "A method for finding structured sparse solutions to nonnegative least squares problems with applications," SIAM Journal on Imaging Sciences, vol. 6, no. 4, pp. 2010-2046, 2013.

[24] P. D. Tao and L. T. An, "Convex analysis approach to d.c. programming: theory, algorithms and applications," Acta Mathematica Vietnamica, vol. 22, no. 1, pp. 289-355, 1997.

[25] Z. Lin, R. Liu, and Z. Su, "Linearized alternating directional method with adaptive penalty for low-rank representation," in Advances in Neural Information Processing Systems, pp. 612-620, 2011.

[26] M.-J. Lai, Y. Xu, and W. Yin, "Improved iteratively reweighted least squares for unconstrained smoothed $\ell_{q}$ minimization," SIAM Journal on Numerical Analysis, vol. 51, no. 2, pp. 927-957, 2013.

[27] X. He, J. Liang, X. Wang et al., "Sparse reconstruction for quantitative bioluminescence tomography based on the incomplete variables truncated conjugate gradient method," Optics Express, vol. 18, no. 24, pp. 24825-24841, 2010.

[28] A. D. Zacharopoulos, P. Svenmarker, J. Axelsson, M. Schweiger, S. R. Arridge, and S. Andersson-Engels, "A matrix-free algorithm for multiple wavelength fluorescence tomography," Optics Express, vol. 17, no. 5, pp. 3025-3035, 2009.

[29] J. A. Tropp, "Greed is good: algorithmic results for sparse approximation," IEEE Transactions on Information Theory, vol. 50, no. 10, pp. 2231-2242, 2004.

[30] B. Dogdas, D. Stout, A. F. Chatziioannou, and R. M. Leahy, "Digimouse: a 3D whole body mouse atlas from CT and cryosection data," Physics in Medicine and Biology, vol. 52, no. 3, pp. 577-587, 2007.

[31] F. Gao, H. Zhao, Y. Tanikawa, and Y. Yamada, "A linear, featured-data scheme for image reconstruction in time-domain fluorescence molecular tomography," Optics Express, vol. 14, no. 16, pp. 7109-7124, 2006.

[32] M. A. Naser and M. S. Patterson, "Improved bioluminescence and fluorescence reconstruction algorithms using diffuse optical tomography, normalized data, and optimized selection of the permissible source region," Biomedical Optics Express, vol. 2, no. 1, pp. 169-184, 2011.

[33] G. Alexandrakis, F. R. Rannou, and A. F. Chatziioannou, "Tomographic bioluminescence imaging by use of a combined optical-PET (OPET) system: a computer simulation feasibility study," Physics in Medicine \& Biology, vol. 50, no. 17, pp. 42254241, 2005. 

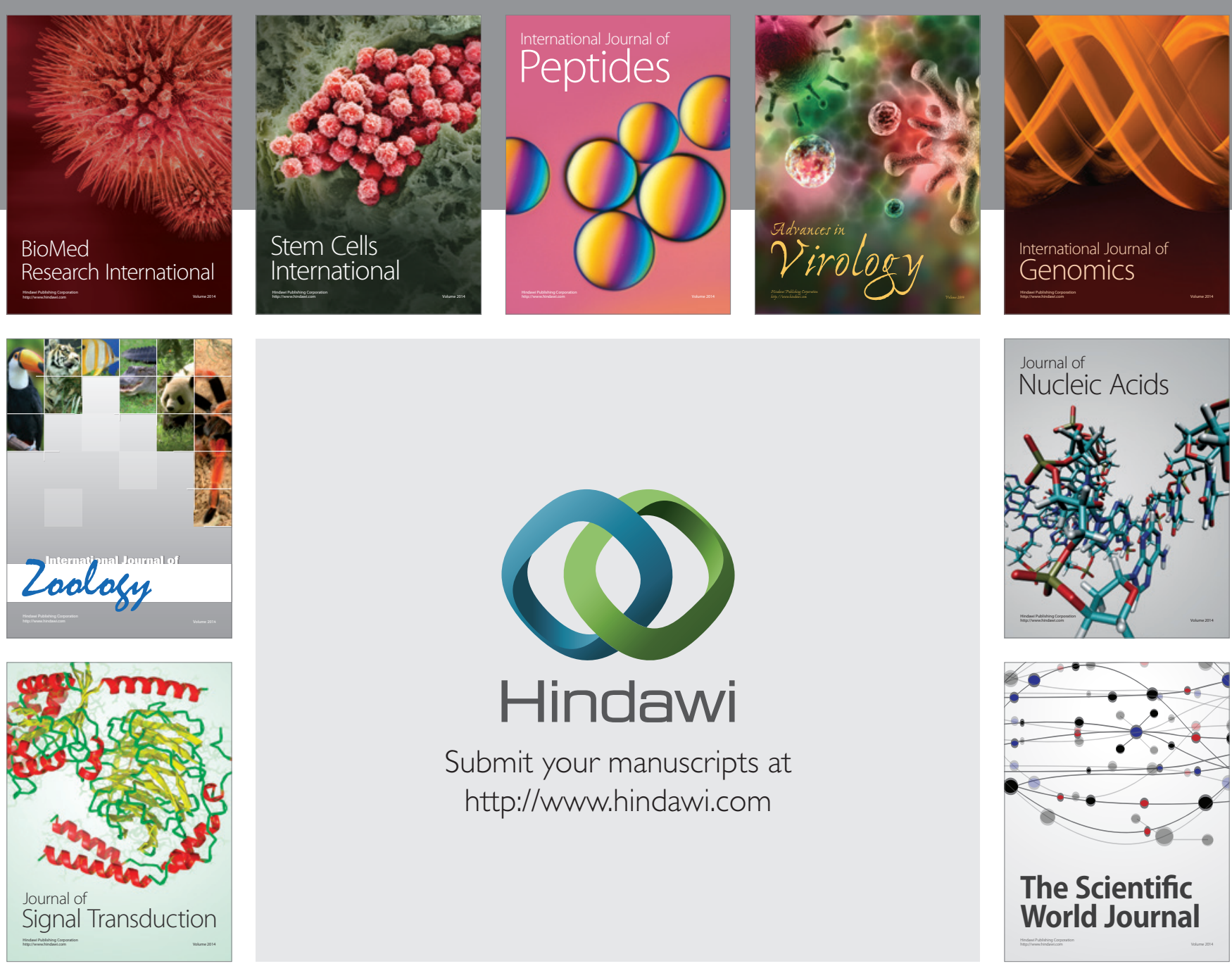

Submit your manuscripts at

http://www.hindawi.com
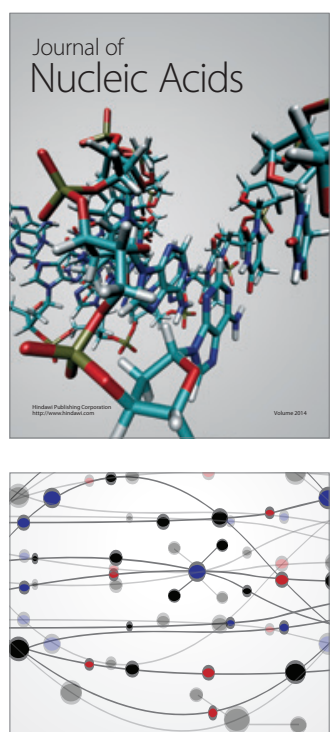

The Scientific World Journal
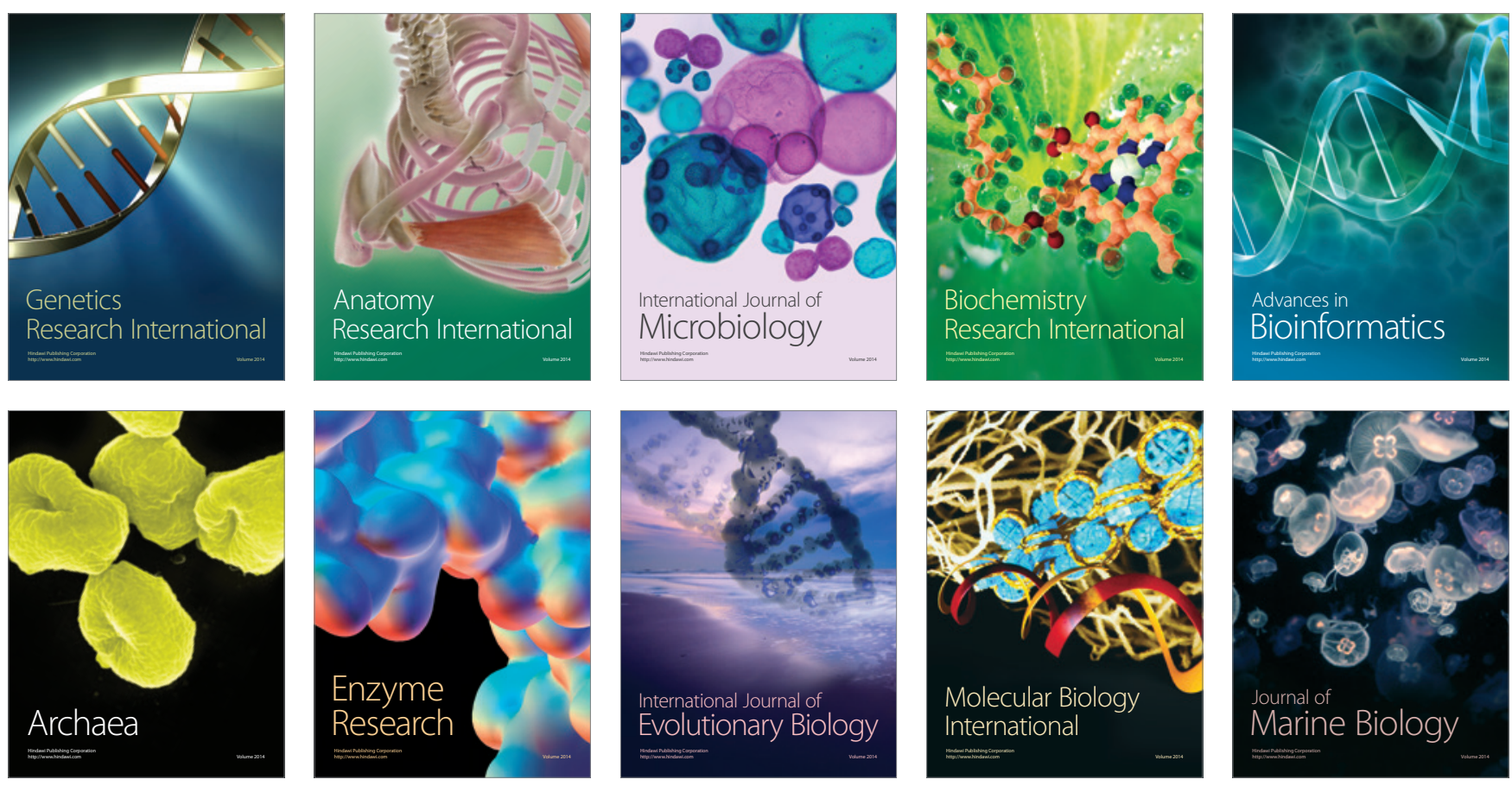